\title{
BRF Negatively Regulates Thermotolerance Defect of fes1a in Arabidopsis
}

\author{
Can $\mathrm{Fu}^{1+}$, Xiaxia $\mathrm{Liu}^{1 \dagger}$, Xuezhi $\mathrm{Li}^{1+}$, Panfei Huo ${ }^{1}$, Jingjing $\mathrm{Ge}^{1}$, Yanfei Hou ${ }^{1}$, \\ Wenwen Yang ${ }^{1}$, Jingxia Zhang ${ }^{2}$, Limin Zhang ${ }^{1}$, Dazhong Zhao ${ }^{3}$, Changle $\mathrm{Ma}^{1 *}$ \\ and Jian Liu ${ }^{1 *}$ \\ ${ }^{1}$ College of Life Sciences, Shandong Normal University, Jinan, China, ${ }^{2}$ Cotton Research Center, Shandong Academy of \\ Agricultural Sciences, Jinan, China, ${ }^{3}$ Department of Biological Sciences, University of Wisconsin-Milwaukee, Milwaukee, WI, \\ United States
}

OPEN ACCESS

Edited by:

Jianhua Zhu,

University of Maryland,

United States

Reviewed by:

Yee-yung Charng,

Academia Sinica,

Taiwan

Ching-Hui Yeh,

National Central University,

Taiwan

*Correspondence:

Changle Ma

machangle@sdnu.edu.cn

Jian Liu

lijsd2002@aliyun.com

${ }^{\text {t}}$ These authors have contributed equally to this work

Specialty section: This article was submitted to

Plant Abiotic Stress,

a section of the journal

Frontiers in Plant Science

Received: 07 October 2019 Accepted: 05 February 2020

Published: 10 March 2020

Citation:

Fu C, LiuX, LiX, Huo P, Ge J, Hou Y, Yang $W$, Zhang J, Zhang L, Zhao $D$, Ma C and Liu J (2020) BRF Negatively Regulates Thermotolerance Defect of fes 1a in Arabidopsis.

Front. Plant Sci. 11:171. doi: 10.3389/fpls.2020.00171
FES1A is a heat shock protein 70 binding protein. Mutation of FES1A leads to a defect in thermotolerance of Arabidopsis; however, independent fes 1a mutants exhibit a range in the extent of thermosensitivity. Here, we found that BRF2, a gene adjacent to FES1A and encoding a component of transcription factor IIIB, affects the thermosensitivity of fes $1 \mathrm{a}$ mutants. Knockout of BRF2 suppressed fes1a thermosensitivity, while overexpression of BRF2 increased thermosensitivity of fes1a. BRF2 in fes1a mutants regulates the transcriptional strength of RNA Polymerase II and accumulation of heat shock proteins and eventually affects the thermotolerance of fes $1 a$. There is a cross-talking between RNA Pol III and Pol II. The cross-talking is initiated by BRF, magnified by the mutation of FES1A, and finally has an effect on thermotolerance.

Keywords: Arabidopsis thaliana, BRF, FES1A, RNA Polymerase II, RNA Polymerase III, heat stress

\section{INTRODUCTION}

Plants are sessile organisms. To cope with high-temperature stress, plants have evolved various adaptive mechanisms to optimize their growth and development to ultimately achieve successful reproduction. Thermotolerance in plants is associated with a number of regulatory and functional genes which constitute complex signaling pathways. It has been found that abscisic acid, salicylic acid, ethylene, reactive oxygen species, membrane fatty acid composition, ubiquitin, heat shock proteins (HSPs), programmed cell death, and photooxidation are all involved in thermotolerance.

Out of numerous heat response-related factors, HSPs are the best understood. HSPs function as molecular chaperones to prevent cellular proteins from denaturation and to promote the refolding of damaged proteins. During heat stress, accumulation of HSPs confers plant cells with acquired thermotolerance, which enables plants to survive under severe heat stress. While acquiring thermotolerance, different classes of HSPs function in different ways. For example, HSP101 is mainly responsible for dispersing the aggregates of heat-denatured proteins (Merret et al., 2017). Cytosolic HSP90 regulates the heat shock response (Mclellan et al., 2007). Once HSP90 chaperon complex becomes inactivated, the Arabidopsis mutant shows a defect in thermotolerance (Fernández-Bautista et al., 2018). HSP70 is a multifunctional factor. Apart from preventing the aggregation of denatured proteins and assisting the refolding of heat-denatured proteins 
(Saibil, 2013; Mayer and Gierasch, 2019), HSP70 interacts with HSFs, cochaperones, and other proteins to coordinate complex signaling networks (Zuiderweg et al., 2017). Disruption of HSP70 function decreases plant thermotolerance ( $\mathrm{Su}$ and $\mathrm{Li}, 2008$ ), while overexpression of HSP70 confers multiple abiotic stress tolerance in transgenic Arabidopsis thaliana (Masand and Yadav, 2016).

HSP70 contains a highly conserved N-terminal nucleotidebinding domain (NBD) and a substrate-binding domain (SBD). ATPase activity is contained in the NBD. In the ATP-bound state, the SBD of HSP70 loosely associates with its substrates (Gowda et al., 2018). Once ATP is hydrolyzed into ADP, the SBD changes conformation and tightly binds to its substrate. When ADP is released from the NBD, HSP70 liberates its substrate and folds back into the natural state to start its next catalytic cycle. In the cycle of HSP70 function, the disassociation of ADP from HSP70 is a rate-limiting step that is accelerated by nucleotide exchange factors (NEFs) (Dragovic et al., 2006). FES1 is a Cytosolic NEF. In addition, FES1 is involved in degradation of misfolded cytosolic proteins (Gowda et al., 2013; Gowda et al., 2016). Arabidopsis FES1A is a HSP70 binding protein and is able to complement the heat-sensitive defect of the Saccharomyces cerevisiae fes $1 \Delta$ strain. In Arabidopsis, knockout of FES1A results in an increase in heat-stress susceptibility (Zhang et al., 2010).

Eukaryotic genes and RNAs are transcribed by three RNA polymerases. RNA polymerase $(\mathrm{Pol}) \mathrm{I}$ and $\mathrm{Pol}$ II are responsible for transcribing rRNAs and mRNAs, respectively, while RNA Pol III transcribes tRNAs, 5S ribosomal RNAs, 7SL RNAs, RNase P, and U6 spliceosomal small nuclear RNAs (Dieci et al., 2013; Duttke, 2014). Genes transcribed by RNA Pol III are involved in ribosome biogenesis, RNA processing, and chromatin regulation (Willis and Moir, 2018). RNA Pol III is a large complex (Hoffmann et al., 2016), in which transcription factor IIIB (TFIIIB) is a central transcription initiation unit. TFIIIB is composed of three proteins: TATA-binding protein (TBP), TFIIIB-related factor (BRF), and B-double prime 1 (BDP1) (Khoo et al., 2014). Yeast BRF1 is involved in thermotolerance (Conesa et al., 2005). Redox sensing by BRF2 modulates resistance to oxidative stress in normal and cancer cells (Gouge et al., 2015). However, no research on plant BRF in stress tolerance was presented.

In our previous studies, two T-DNA insertion mutants of fes $1 a$ exhibited defects in thermotolerance but to different degrees (Zhang et al., 2010). In this study, three independent fes1a T-DNA insertion mutants were used to evaluate the mechanisms of thermotolerance. Interestingly, three fes $1 a$ mutants exhibited different extents of thermo-sensitivity. Using allelic hybridization and evaluation of double mutants, we found that the factor suppressing the thermosensitivity of fes $1 a$ mutants was BRF2, a gene adjacent to FES1A, which encodes a component of TFIIIB. Further analysis showed that the amount of BRF2 in the absence of FES1A affected the activity of RNA Pol II, which ultimately regulates the response of thermotolerance-related molecules, such as HSPs, and modulates the thermotolerance in fes1a. These results provide important evidence that BRF of the Pol III in plant regulates thermotolerance.

\section{MATERIALS AND METHODS}

\section{Plant Material and Growth Conditions}

Arabidopsis thaliana mutants (Supplemental Table 1) were obtained from the ABRC (http://abrc.osu.edu). T-DNA insertions were confirmed using PCR and sequencing (Supplemental Table 2). After backcrossing twice with WT, the mutants were used for the experiments. The thermotolerance of seedlings was evaluated according to the intermittent temperature-increasing regime. First, ten-day-old plants were acclimated at $38^{\circ} \mathrm{C}$ for $2 \mathrm{~h}$ and then subjected to lethal heat treatment at $45^{\circ} \mathrm{C}$ for different time periods. Following that, the plants were transported to a growth chamber under normal growth conditions for recovery. The phenotypes were photographed after seven days of recovery (Zhang et al., 2010).

\section{Yeast Complementation Assay}

The recombinant yeast strains and plasmids used in this study are listed in Supplemental Table 3. The primers used are shown in the Supplemental Table 4. The ScBRF1- $\triangle N 10$ cDNA fragment (lacking the coding sequence for the first 10 amino acids) was cloned into pYX242WS (Gibson, 2011; Wang et al., 2013), generating the plasmid $S c B R F 1-\Delta N 10$-pYX. The Arabidopsis BRF2 cDNA sequence was ligated into plasmid pJFE3 (Shen et al., 2012) to generate the plasmid AtBRF2pJFE3. The deletion of $S c B R F 1$ was generated via the Cre-loxP method (Hegemann et al., 2006).

\section{Generation of Arabidopsis Transgenic Plants}

To generate the $B R F 2$ overexpression construct, the open reading frame of BRF2 was amplified by PCR (Supplemental Table 5) and then inserted into the $A f l$ II site of plasmid pMY72, resulting in the plasmid BRF2-(HA) $\times 2$-pMY72. The PCR-amplified fusion fragment of BRF2-(HA) $\times 2$ (Supplemental Table 5) was inserted into the EcoR I and Kpn I sites of $P_{\text {LeHsp23.8-pRT101 (Yi et al., }}$ 2006), generating the plasmid $P_{\text {LeHsp23.8 }}$ BRF2-HA $A_{2}$-pRT101. Arabidopsis was transformed using the floral dipping method (Clough and Bent, 1998).

The BRF2 gene in the fes 1a background was mutated using CRISPR/Cas9 (Xing et al., 2014). The BRF2-CRISPR/Cas9 cassette was generated by PCR amplification from pCBCD1T1. The PCR products were digested with Bsa I and ligated into pHSE401, generating the plasmid BRF2-pHSE401 that was used for Arabidopsis transformation. The transgenic lines were selected using hygromycin B and further confirmed by enzyme digestion and sequencing. All primers used are listed in Supplemental Table 5.

\section{Subcellular Localization of BRF2}

The cDNA of BRF2 was amplified by PCR (Supplemental Table 5) and then inserted into the $P_{35 \mathrm{~S} C a M V^{-}} G F P$ vector, generating the fusion construct $P_{35 s C a M V}-B R F 2-G F P$. The fusion construct was transformed into Arabidopsis by the floral dipping method. The subcellular localization of the BRF2-GFP fusion was 
examined by confocal laser scanning microscopy (Leica TCS SP8).

\section{RNA Extraction, RT-PCR, and qRT-PCR}

Total RNA was isolated from 15-day-old seedlings using TRIzol reagent (Invitrogen). First-strand cDNA was synthesized using a PrimeScript ${ }^{\mathrm{TM}}$ RT Reagent Kit with a gDNA Eraser (Takara). RTPCR was performed using the primers shown in Supplemental Table 6. qRT-PCR was performed on a Roche LightCycler ${ }^{\circledR} 480$ (LC480) using the SYBR Green system. The gene specific primers used in the qRT-PCR analysis are listed in Supplemental Table 7. The ACTIN8 gene was used as an internal control.

\section{Immunoblotting Analysis}

Western blot was performed as described previously (Zhang et al., 2010) using the primary antibodies anti-FES1A antibody, anti-HSC70 monoclonal antibody (SPA-817; Stressgen, San Diego, CA, US), anti-HSP101 N-terminal (AS07 253; Agrisera, Vännäs, Sweden), anti-CLPB N-terminal (Yang et al., 2006), anti-small HSP class II antibody (AS07 255; Agrisera), antiHSP21 antibody (AS08 285; Agrisera), anti-ACTIN antibody (AB10007; Sangon, Shanghai, China), anti-HISTONE 3 antibody (AS10 710; Agrisera), anti-HA antibody (Ab9110; Abcam), antiS2P-CTD (ab5095, Abcam), and anti-S5P-CTD (ab5131,
Abcam) antibodies. Anti-BRF antibody (rabbit) was generated using the C-terminal fragment of BRF2 (361-604 amino acids).

\section{Statistical Analysis}

All experiments in this study were performed at least three times, and the values are expressed as the mean \pm SD. Statistical significance was determined by Duncan's multiple range test $(P<0.05)$.

\section{RESULTS}

\section{The fes1a Mutants Exhibit Differential Sensitivities to Heat Stress}

In our previous study, we examined the thermotolerance of two allelic mutants of fes $1 a$, each of which contained a T-DNA insertion in the sixth exon of FES1A (Zhang et al., 2010). Both mutants showed defects in thermotolerance, but to significantly different degrees. To investigate the cause of variability in thermotolerance of the fes1a mutants, we chose three allelic mutants for further thermotolerance evaluation (Figure 1). Sequencing results revealed that cs842189 had a T-DNA insertion in the second exon, while salk_012416 in the fifth intron and salk_072075 harbored a T-DNA insertion in the sixth exon (Figure 1A; Supplemental Figure 1). To

A

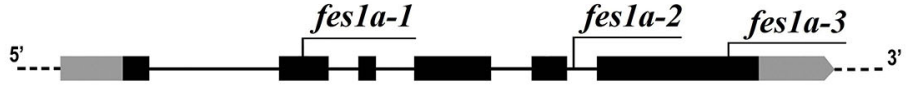

B

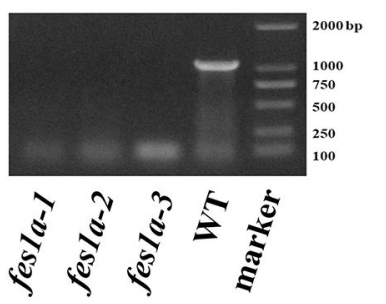

C

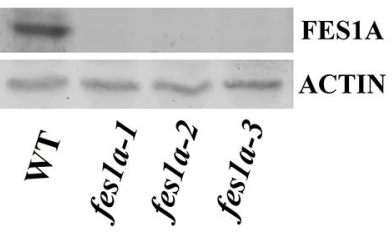

D

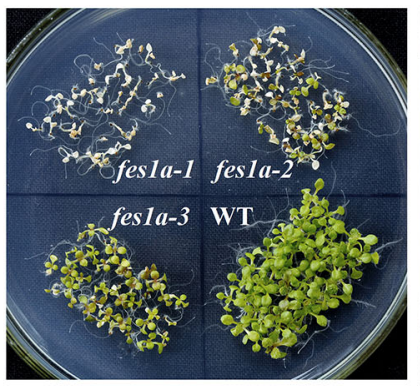

$38^{\circ} \mathrm{C} / 2 \mathrm{~h} \rightarrow 45^{\circ} \mathrm{C} / 2 \mathrm{~h}$

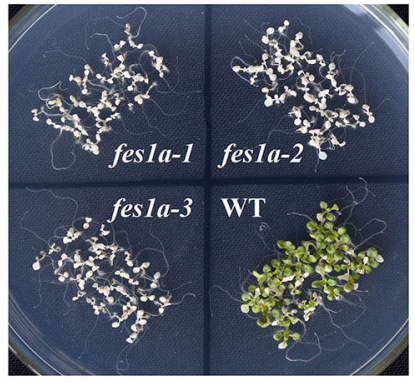

$38^{\circ} \mathrm{C} / 2 \mathrm{~h} \rightarrow 45^{\circ} \mathrm{C} / 3.5 \mathrm{~h}$

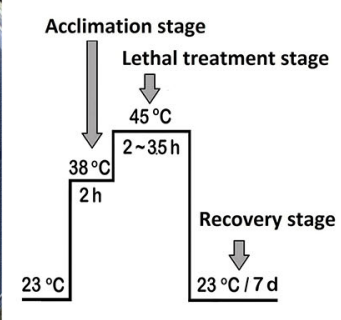

FIGURE 1 | Differences in thermotolerance of the three fes1a mutants. (A) A diagram showing T-DNA insertions in three examined fes1a mutants. Exons are black boxes, untranslated coding regions are grey boxes, and introns are lines. RT-PCR (B) and western blots (C) exhibiting transcription and translation of FES1A, respectively. Plants were treated at $38^{\circ} \mathrm{C}$ for $2 \mathrm{~h}$ before total RNA and protein extraction. (D) Evaluation the thermotolerance of fes $1 \mathrm{a}$ mutants. A temperatureincreasing regime for thermotolerance evaluation is schematically shown on the right. Ten-day-old plants were first acclimated at $38{ }^{\circ} \mathrm{C}$ for $2 \mathrm{~h}$ and then subjected to lethal heat treatment at $45^{\circ} \mathrm{C}$ for $2 \sim 3.5 \mathrm{~h}$ as indicated in the center of each petri dish. After heat stress, plants were transferred to a growth chamber under normal growth conditions for recovery and were photographed after seven days of recovery. 
distinguish these three fes $1 a$ mutants, we sequentially renamed them fes $1 a-1$ to fes $1 a-3$ based on the T-DNA locations from $5^{\prime}$ - to $3^{\prime}$-UTR (Figure 1A, Supplemental Table 1).

RT-PCR and western blot showed that the FES1A transcript and FES1A protein were absent from all three fes $1 a$ mutants (Figures 1B, C), suggesting that these fes $1 a$ mutants are loss-of-function mutants. T-DNA insertions in different sites did not result in the formation of any truncated FES1A proteins, since no small FES1A fragments was detected in any of the fes $1 a$ mutants using the anti-FES1A antibody (Supplemental Figure 2), which recognizes both the N-terminal and the C-terminal of FES1A (Zhang et al., 2010).

We evaluated the heat sensitivities of all three fes $1 a$ mutants. Ten-day-old seedlings were first subjected to heat acclimation at $38^{\circ} \mathrm{C}$ for $2 \mathrm{~h}$, and then exposed to lethal heat-stress at $45^{\circ} \mathrm{C}$ for different lengths of time. Upon $2 \mathrm{~h}$ of lethal stress at $45^{\circ} \mathrm{C}$, fes $1 \mathrm{a}-1$ and fes $1 a-2$ both exhibited symptoms of severe damage (Figure 1D), while fes $1 a-3$ displayed great resistance to heat stress. When the lethal heat-stress was extended to $3.5 \mathrm{~h}$, all three fes $1 \mathrm{a}$ mutants, but not the wild-type (WT) plants, finally perished, indicating that all fes1a mutants possessed thermotolerance defects, but with different sensitivities. In addition, the survival rates of the seedlings undergoing an acquired thermotolerance challenge indicated that fesla-3 had the best thermotolerance among the three fes $1 a$ mutants, with the survival rate reaching $92.5 \%$, while fes $1 a-2$ and fes $1 a-1$ had $41.7 \%$ and $0.8 \%$ survival, respectively (Supplemental Figure 3A). These phenotypes (Figure 1D) and physiological data (Supplemental Figure 3) confirmed our previous results that the null mutants of FES1A exhibited differential extents of thermotolerance (Zhang et al., 2010).

\section{The Differential Thermotolerance of fes1a Mutants Is Closely Linked With Their Adjacent BRF2 Gene}

Although fes1a-3 phenotype exhibited its significantly higher thermotolerance than those of fes $1 a-1$ and fes $1 a-2$, we clearly observed the thermotolerant difference between fesla-1 and fes1a-2. In other words, fes $1 a-2$ was more tolerant than fes $1 a-1$ (Figure 1D, Supplemental Figure 3A). Accordingly, the factor modulating the thermotolerance should be ubiquitous in all the three fes 1 a mutants. A simple explanation for the observation was that the involved factor might genetically link to FES1A locus. Thus, we performed two sets of allelic hybridization experiments, in which three independent lines of fesla were included. After selecting out homozygous F2 progenies, more than 100 homozygous F2 progenies were evaluated for thermosensitivity. As a result, the thermotolerance of F2 progenies was exactly determined by the genotype of the T-DNA insertion. In other words, if the genotype of the T-DNA insertion in an F2 individual was the same as its parent line, the parent and the offspring showed identical thermotolerance (Supplemental Table 8). Therefore, this suggests that the factor differentiating the thermo-sensitivities of the fes 1 a mutants was closely linked to the FES1A locus or to the individual location of T-DNAs. As each of the T-DNAs in fes $1 a$ could exert a significant effect on the thermo-sensitivities of the fes $1 a$ mutants, the genes possibly influenced by the T-DNA insertions should be confined to the outer edge of FES1A. Using qRT-PCR, we examined the expression of the proximal genes adjacent to FES1A in the fes 1a mutants. Only BRF2 (Lagrange et al., 2003), which encodes one of the three components of transcription factor IIIB, was significantly downregulated in comparison to WT (Supplemental Figure 4).

In order to investigate whether $B R F 2$ is responsible for regulating thermotolerance in fesla mutants, we knocked out the BRF2 gene using the CRISPR/Cas9 system in the fes $1 a-2$ mutant background (Figure 2). DNA sequencing revealed that the fes1a-2 brf2-ed 1 double mutant had 11 base pairs deletion and 13 base pairs mutated in the tenth exon of BRF2, while fes $1 a$ 2 brf2-ed 2 lost 7 base pairs in the tenth exon of BRF2 (Figure 2A). To determine their thermotolerance, 10-day-old Arabidopsis seedlings were first exposed to $38^{\circ} \mathrm{C}$ for $2 \mathrm{~h}$, then subjected to lethal heat treatment at $45^{\circ} \mathrm{C}$ for $2 \mathrm{~h}$, and finally moved to a room at $23^{\circ} \mathrm{C}$ for 7 days. As shown in Figure $\mathbf{2 B}$ and Supplemental Figure 5, mutations in BRF2 increased the thermotolerance of fes $1 a-2$ and the accumulation of HSPs. Interestingly, the brf2 single mutant did not exhibit any visible change in thermotolerance (Supplemental Figure 6). Arabidopsis $B R F$ gene family has three members, $B R F 1$ (At2g45100), BRF2 (At3g09360), and BRF3 (At2g01280). We further bred the double mutants of BRFs. The homogenous double mutants of brf1 brf3 and brf2 brf3 were generated, while the double mutation of BRF1 and BRF2 led to sterility (Zhang et al., 2019). We determined the thermotolerance of brf1 brf3 and brf2 brf3. Both presented increases of thermotolerance, compared to WT (Supplemental Figure 7), suggesting that BRF not only acts as a suppressor of the fes1a mutant but also negatively regulates the thermotolerance in WT.

We further explored the effect of BRF2 on thermotolerance by overexpressing HA-tagged BRF2 under the control of an HSP promoter in both WT plants and fes1a-2 mutants. Of the $\mathrm{T}_{3}$ transgenic lines, OEBRF2-1/WT and OEBRF2-2/WT, which only had one HA-tagged BRF2 insertion, were selected for further studies. We crossed the OEBRF2/WT lines with fes $1 a-2$ to obtain the allelic OEBRF2-1/fes1a-2 and OEBRF2-2/fes1a-2 lines. Western bolt found that BRF2 was overexpressed in transgenic lines (Figure 2C). BRF2 overexpression lines were subjected to $38^{\circ} \mathrm{C}$ for $2 \mathrm{~h}$ acclimation, then $45^{\circ} \mathrm{C}$ for $1.5 \mathrm{~h}$ lethal heat stress, and finally left at $23^{\circ} \mathrm{C}$ for 7 days. We found that overexpression of BRF2 led to increased susceptibility of fes $1 a-2$ to heat stress, but had no effect on the WT background (Figure 2D). Taken together, our results show that the abundance of BRF2 in fes1a mutants was correlated with the sensitivity of fes $1 a$ mutants to heat stress, further suggesting that $B R F 2$ is a negative regulator of thermotolerance defect in fes1a.

$B R F 1, B R F 2$, and $B R F 3$ may have similar functions. To test this speculation, fesla-1 brf1 and fes1a-1 brf3 double mutants were generated by crossing fes $1 a-1$ with $b r f 1$ and brf3 T-DNA insertion mutants (Figure 2E), respectively, and the thermotolerance of the double mutants was evaluated. As shown in Figure 2F, knockout of $B R F 1$ or BRF3 significantly increased the thermotolerance of $f e s 1 a-1$. Therefore, our results suggest that all three $B R F$ genes have similar 

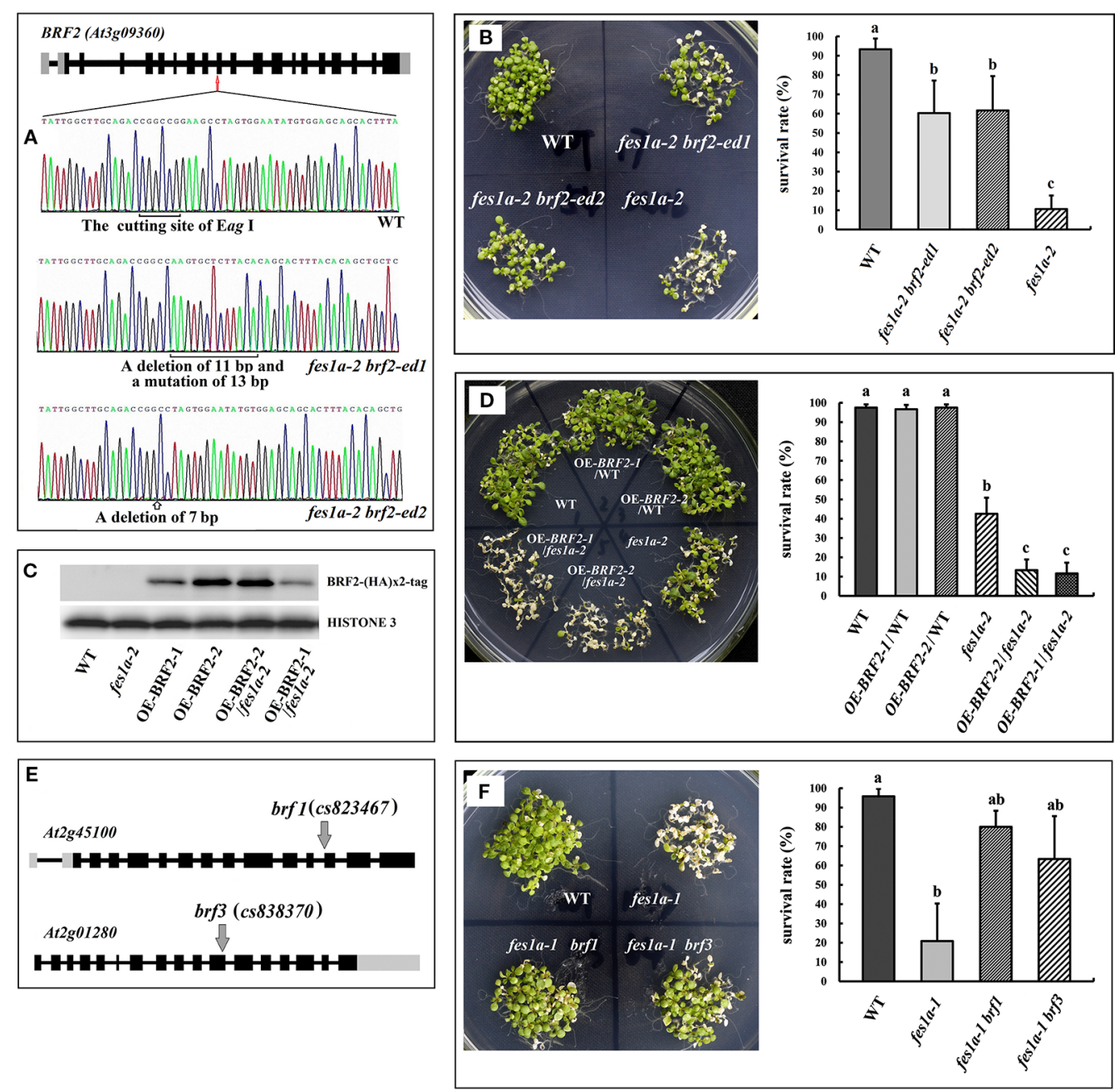

FIGURE 2 | BRF genes suppress the thermosensitivity of fes1a mutants. (A) Sequencing results revealed CRISPR/Cas9 generated mutations (at $10^{\text {th }}$ exon) in the BRF2 gene in fes1a-2 brf2-ed1 and fes1a-2 brf2-ed2 double mutants. (B) The fes1a-2 brf2-ed1 and fes1a-2 brf2-ed2 mutants showing enhanced thermotolerance in comparison with the fes1a-2 mutant (left). The corresponding survival rate is on the right. (C) Western blot result displaying expression of the BRF2-(HA) $\times 2$ protein in overexpression transgenic lines. HISTONE 3 was used as a loading control. (D) Overexpression of BRF2 decreased the thermotolerance of the fes $1 a-2$ mutant, but had no effect on wild-type (left). The corresponding survival rate is on the right. (E) A diagram illustrating T-DNA insertions in BRF1 and BRF3 gene. (F) Mutation in BRF1 and BRF3 genes improved thermotolerance in the fes 1a-1 mutant (left). The corresponding survival rate is on the right. Data in (B, D, F) represent the means of 3 replicates \pm SD. For each column, different letters $a, b, c$ indicate significant differences at $P<0.05$. The temperature regime of heat stress in (B, D, F) was the same as shown in Figure 1, except that the lethal treatment at $45^{\circ} \mathrm{C}$ lasted for $2 \mathrm{~h}$ in (B), $1.5 \mathrm{~h}$ in (D), and $2 \mathrm{~h}$ in (F).

functions in regulating the heat sensitivity of fesla mutants.

We determined the level of BRF2 in fes $1 a-1$ and fes $1 a-3$ by using the anti-BRF2 antibody that identified BRF2 protein well in spite of a very faint nonspecific background signal included (Supplemental Figure 8). Western blot showed the amount of BRF2 in fes 1a-1 was higher than that in fes 1a-3 (Figure 3), which supported the suggestion that BRF2 negatively regulated thermotolerance in fes $1 a$ mutants.

\section{Arabidopsis BRF2 Is a Functional Ortholog of Yeast BRF1}

Phylogenetic analysis found that BRFs from yeasts, plants, and animals clustered together, suggesting that they all evolved from a common ancestor (Supplemental Figure 9, Supplemental Table 9). Subcellular localization analysis showed that the fusion protein BRF2-GFP was localized in the nucleus (Figures 4A-C). To investigate whether BRF2 is a functional ortholog of Saccharomyces cerevisiae BRF1, we performed yeast functional complementation experiments. As knock-out of ScBRF1 is fatal (Colbert and Hahn, 1992), we adopted an alternative procedure to obtain a ScBRF1 conditional knockout mutant. The W303a strain was first transformed with the plasmid $S c B R F 1-\Delta N_{10}$-pYX (Supplemental Table 3), in which open reading frame of $S c B R F 1$ was deleted $30 \mathrm{bp}$ from the start codon. Subsequently, the ScBRF1 locus was knocked out to generate $S c B R F 1 \Delta p S c B R F 1-\triangle N_{10}$ strain. Under normal conditions, $S c B R F 1 \Delta p S c B R F 1-\triangle N_{10}$ strain grew, 


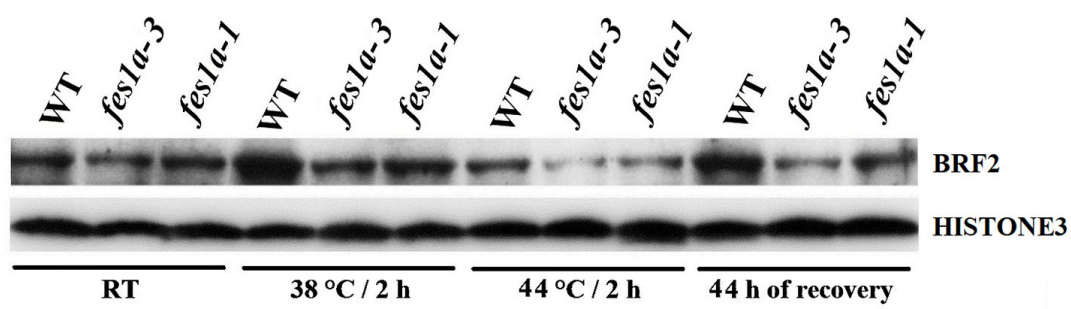

FIGURE 3 | Western blot results exhibiting the expression of BRF2 protein in 10-day-old seedlings of WT and fes1a mutants.
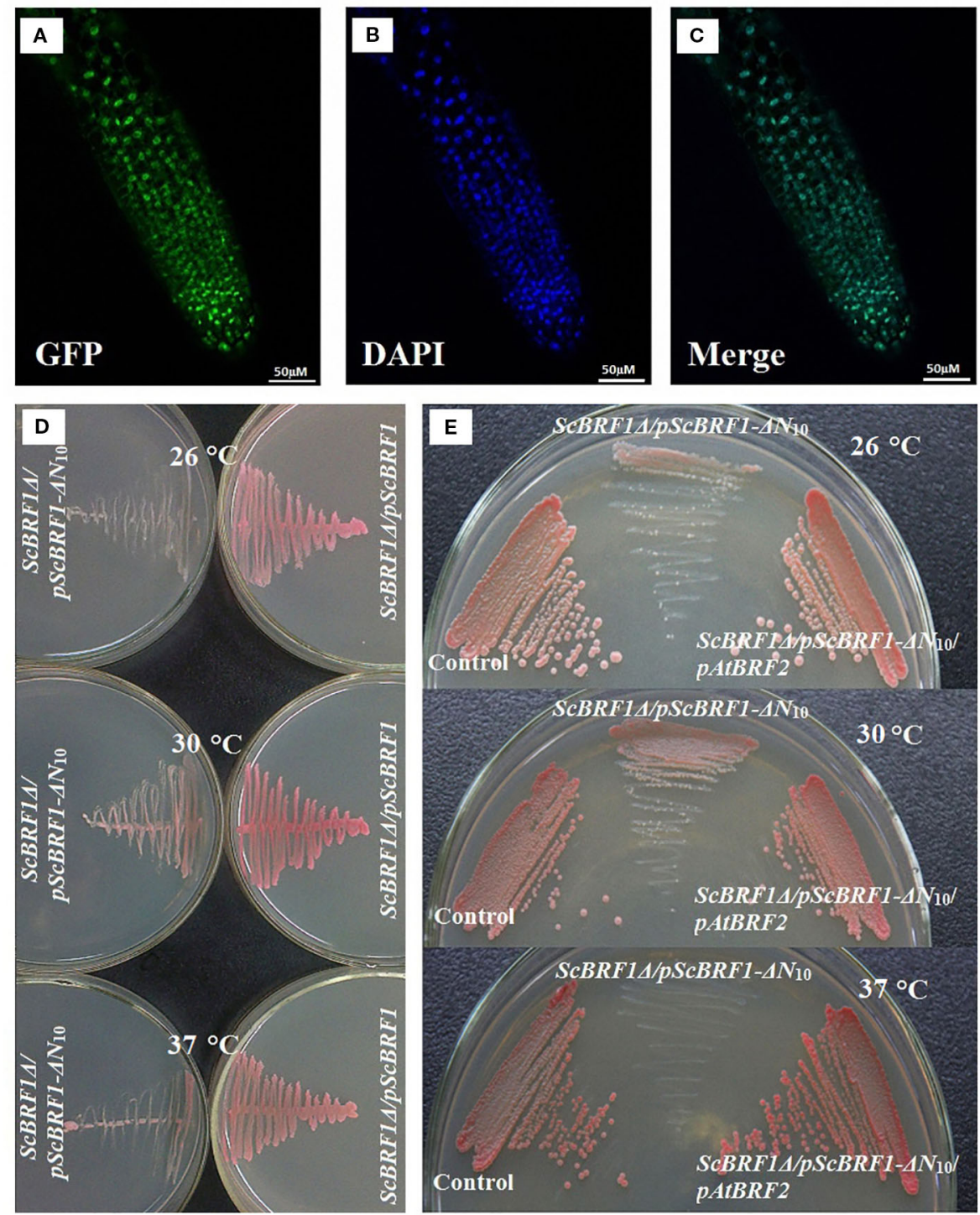

FIGURE 4 | Arabidopsis BRF2 complements the thermotolerance defect of S. cerevisiae brf1 mutant. (A-C) Confocal images illustrating subcellular localization of the BRF2-GFP fusion protein in Arabidopsis roots. The green fluorescent image of BRF2-GFP fusion (A) and the blue fluorescent of nucleus stained with DAPI (B) are merged into (C). (D) The substitution of ScBRF1 with an N-terminal truncated fragment (harbored in pScBRF1- $\Delta$ N10 plasmid) resulted in defect in growth, especially at adverse temperatures. (E) The susceptibility of ScBRF1 $\Delta / \mathrm{pScBRF} 1-\Delta \mathrm{N} 10$ strain to adverse temperatures was remedied by overexpression of the Arabidopsis BRF2. W303 strain with pJFE3 and pYX242WS vectors was used as a control. 
albeit slowly (Figure 4D). Under high and low temperature stresses, the growth of strain $S c B R F 1 \Delta p S c B R F 1-\Delta N_{10}$ was further severely suppressed (Figure 4D). This defect in thermotolerance was rescued by expressing $A t B R F 2$ in $S c B R F 1 \triangle p S c B R F 1-\triangle N_{10}$ strain (Figure 4E, Supplemental Table 3), suggesting that the Arabidopsis BRF2 is a functional ortholog of yeast BRF1.

\section{At the Recovery Stage, HSP Accumulation Is Different Between the fes1a Mutants}

Of the three fes $1 a$ mutants examined in this study, fes $1 a-1$ and fes $1 a-$ 3 were the most and least sensitive to heat stress, respectively, although fes $1 a-1$ showed slightly sensitive to heat stress, compared to fes $1 a-2$. We chose fes $1 a-1$ and fes $1 a-3$ to detect protein expression changes in response to heat stress. Western blot results (Figure 5) showed that all examined HSPs, including HSP101, CLPB, HSP90, HSP70, HSP21, and small HSP class II were at similar levels in fes1a1 and fes $1 a-3$ after heat acclimation at $38^{\circ} \mathrm{C}$ for $2 \mathrm{~h}$, which hardly explain the differential thermotolerance of these two fes $1 a$ mutants. Therefore, we further determined the levels of HSP proteins over 48 $\mathrm{h}$ of the recovery period. In general, the levels of the HSPs at the recovery stage were in the order of WT $>$ fes $1 a-3>$ fes $1 a-1$, and the differences were significant, especially as for HSP101, CLPB, HSP70, and small HSP (Figure 5). These results suggest that accumulation of

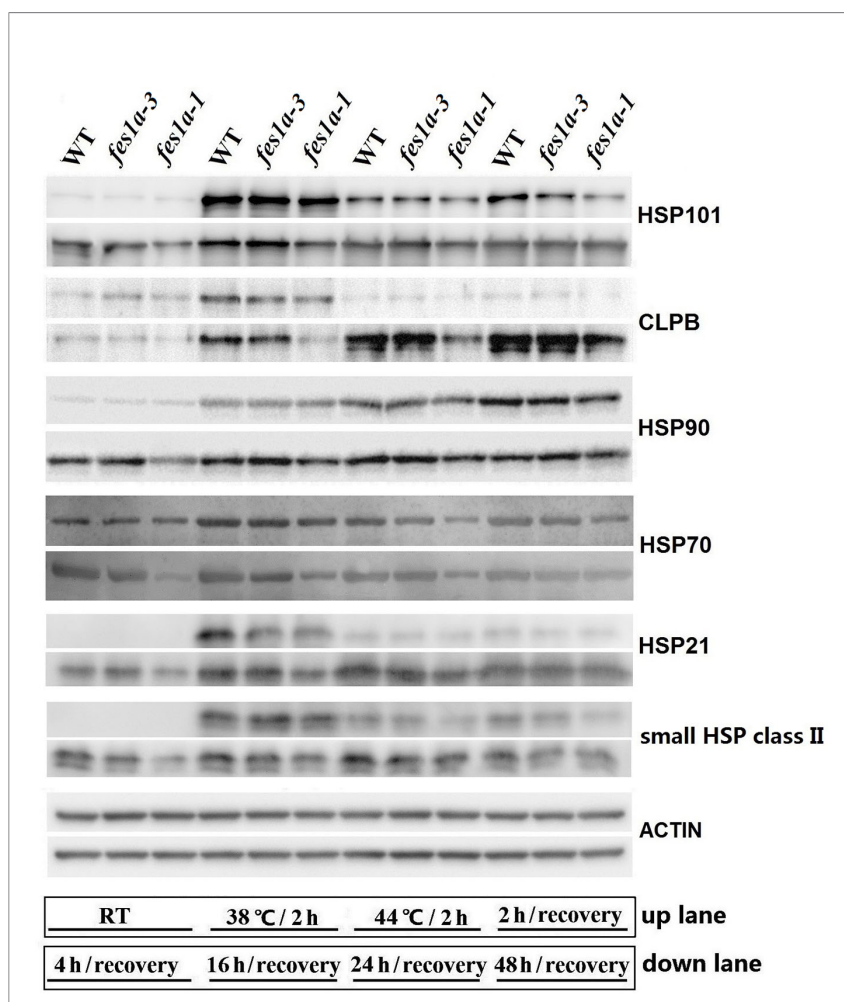

FIGURE 5 | The expression of HSPs over the period from heat acclimation to the stage of recovery. Ten-day-old seedlings were first acclimated for $2 \mathrm{~h}$ at $38^{\circ} \mathrm{C}$, and then exposed to $44^{\circ} \mathrm{C}$ for $2 \mathrm{~h}$, and lastly returned to room temperature (RT) for recovery. At the indicated times (shown at the bottom of the picture), proteins were extracted for western blots. ACTIN was used as a loading control.
HSPs at the recovery stage was well correlated with the differential thermotolerance among WT, fes $1 a-1$, and fes1a-3.

\section{The Mutant of fes1a-3 Presented Higher Activity of RNA Pol II than fes1a-1}

According to the different expressions of HSPs at the recovery stage, we further evaluated the transcriptional activity of RNA Pol II in fesla-1 and fesla-3 by examining the phosphorylation of the Cterminal domain (CTD) of RNA Polymerase II largest subunit 1 (RPB1), using anti-S2P-CTD and anti-S5P-CTD antibodies. The CTD of RPB1 is composed of tandem heptad repeats that are critical for the activity of RNA Pol II (Hsin and Manley, 2012). The phosphorylation status of Ser2 and Ser5 in the CTD of RPB1 could serve as an indicator of RNA Pol II activity. Although lower than in WT, the level of phosphorylated RPB1 CTD in fes1a-3 was higher than in fes $1 a-1$ at the recovery stage after exposure to a severe heat stress (Figure 6). These results confirm that the activity of RNA Pol II increased faster in fes $1 a-3$ than fes $1 a-1$ during recovery from a severe heat shock. Further, we determined the activity of RNA Pol II in WT, brf2, fes $1 a-2$ and fes $1 a-2$ brf2-ed 1 double mutant. The result indicated that the single mutant of $b r f 2$ did not change the activity of RNA Pol II, compared to WT. A mutation of BRF2 in genetic background of fes1a-2, however, increased phosphorylation levels of RPB1 CTD at the recovery stage after a severe heat stress (Supplemental Figure 10), suggesting that BRF2 negatively affects the activity of RNA Polymerase II in fes1a.

\section{DISCUSSION}

In this study, we found that three T-DNA insertion mutants of fes $1 a$ exhibited significantly different heat sensitivities (Figure 1). The closer the T-DNA insertion was towards the 3'UTR of FES1A, the more tolerant the fes1a mutant was to heat stress. Using allelic hybridization (Supplemental Table 8) and evaluation of double mutants, we found that BRF2, a gene downstream of FES1A, is responsible for increasing the thermo-sensitivity of fes1a mutants. Knockout of BRF2 in a fes 1 a mutant rescued the fes 1 a thermotolerance defect, while overexpression of BRF2 in fes1a mutant further increased heat sensitivity (Figure 2). Thus, the lower level of BRF2 in fes1a-3 was suggested to being responsible for its more significant decrease of sensitivity to heat stress (Figure 3) when compared to fes1a-1.

Arabidopsis BRF2 was a negative regulator of RNA pol II transcription in the fes $1 a$ mutant. The amount of BRF2 in fes1a mutants was negatively correlated with the activity of RNA Pol II. RPB1 is the largest subunit of the RNA Pol II complex (Hsin and Manley, 2012). At transcription initiation, Ser2 and Ser5 residues in the CTD of RPB1 are phosphorylated, whereas as transcription nears termination, Ser5-P and Ser2-P are gradually dephosphorylated, regenerating unphosphorylated RPB1 that can be recycled for another round of transcription. Thus, the level of phosphorylated CTD reflects the activity of Pol II transcription. As suggested by a significant increase in the phosphorylated status of Ser2 and Ser5 of the CTD of RPB1 in fes1a-3 (Figure 6), RNA Pol II transcription is more active in fesla-3 than in fesla-1. In addition, knockout of the 


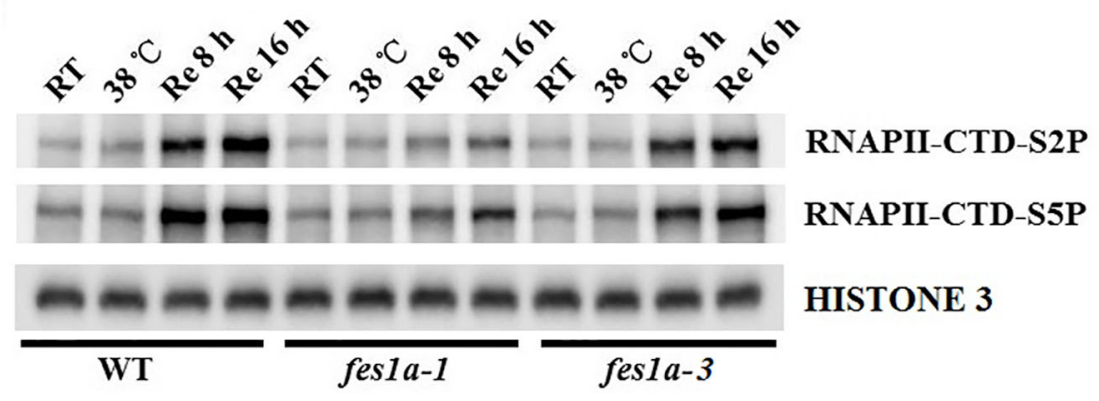

FIGURE 6 | Phosphorylation levels of the C-terminal domain (CTD) of RNA Polymerase (Pol) II largest subunit 1 (RPB1) in fes1a-1 and fes1a-3. Ten-day-old seedlings were acclimated for $2 \mathrm{~h}$ at $38^{\circ} \mathrm{C}$, then exposed to $44{ }^{\circ} \mathrm{C}$ for $2 \mathrm{~h}$, and lastly returned to room temperature for recovery. Seedlings were sampled at indicated times. Phosphorylation levels of RPB1 CTD repeats were determined with RNA Pol II CTD repeat phospho Ser-2 and Ser5 antibodies.

BRF2 increased the extent of phosphorylation of RPB1 in fes1a-2 (Supplemental Figure 10), suggesting BRF2 negatively regulated the strength of Pol II transcription in fes1a. Further, western blot results showed that the accumulation of HSPs in fes $1 a-3$ was higher than in fes1a-1. Therefore, the differential strengths of general transcription and the differential accumulation of HSPs are molecular evidence of significant differences in thermosensitivities between fes $1 a-1$ and fes1a-3. The possible relationships among FES1A, BRF, and thermotolerance-related genes are presented in Figure 7.

It has been shown that the mutation of $S$. cerevisiae $B R F 1$ affected RNA Pol II transcription. Mutations in ScBRF1- $\triangle N 10, S c B R F 1-$ $\triangle N 25$, and $S c B R F 1-\triangle C 50$ all lead to an increased expression of cytochrome c (Colbert and Hahn, 1992), while the yeast mutant ScBRF1-II.6, which has point mutations at D464A and D466A on ScBRF1, enhances mRNA transcription (Conesa et al., 2005). In short, the mutation of ScBRF1 is in favor of Pol II transcription. Our

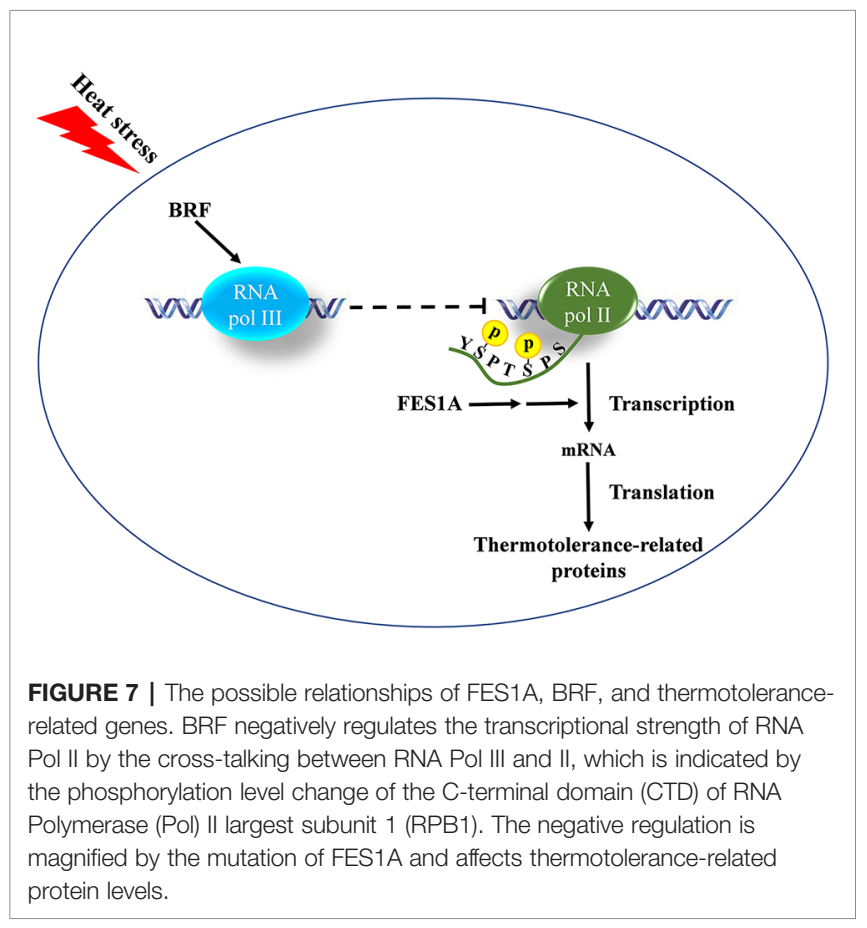

results suggest that Arabidopsis BRF2, a functional ortholog of yeast ScBRF1, negatively affects the activity of RNA Pol II and is involved in the heat response of fesla mutants, indicating there is a crosstalking between RNA Pol III and Pol II, which affects thermotolerance. Further, the negative regulation of the double mutations of BRFs on thermotolerance in WT suggests that the cross-talking is independent of fes $1 a$. The single mutation of BRF affected the thermotolerance in fes $1 a$, but the thermotolerance in WT was regulated by the double mutations of BRFs. In other words, the effect of BRF is magnified in fesla. However, the involved mechanism needs to be studied further.

\section{DATA AVAILABILITY STATEMENT}

The raw data supporting the conclusions of this manuscript will be made available by the authors, without undue reservation, to any qualified researcher.

\section{AUTHOR CONTRIBUTIONS}

CF, XLiu and XLi performed majority of the research. Western blot was performed by CF, XLiu, PH, and JG. WY and JZ performed the phenotyping experiments. Bioinformatic analysis was carried out by XLiu and LZ. JL designed the research and wrote the main manuscript text. JL, CF, CM, and DZ revised the manuscript. All authors read and approved the final manuscript. CF, XLiu, and XLi contributed equally to this work.

\section{FUNDING}

This work was supported by the National Natural Science Foundation of China (31270298, 31470352, 3182004) and the Research Fundamental Capacity Improvement Project for Middle Age and Youth Teachers of Guangxi Universities (2019KY0517). Research in the lab of DZ was supported by the National Science Foundation grant IOS-1322796 and the Research Growth Initiative (RGI). The funder had no role in the study design, data collection and analysis, decision to publish, or preparation of the manuscript. 


\section{ACKNOWLEDGMENTS}

We wish to thank Professor Jian-Kang Zhu for his critical reading of the manuscript as well as Professor Qijun Chen for generously providing the CRISPR-Cas9 system.

\section{REFERENCES}

Clough, S. J., and Bent, A. F. (1998). Floral dip: a simplified method for Agrobacterium-mediated transformation of Arabidopsis thaliana. Plant J. 16, 735-743. doi: 10.1046/j.1365-313x.1998.00343.x

Colbert, T., and Hahn, S. (1992). A yeast TFIIB-related factor involved in RNA polymerase III transcription. Genes Dev. 6, 1940-1949. doi: 10.1101/gad.6.10.1940

Conesa, C., Ruotolo, R., Soularue, P., Simms, T. A., Donze, D., Sentenac, A., et al. (2005). Modulation of yeast genome expression in response to defective RNA polymerase III-dependent transcription. Mol. Cell. Biol. 25, 8631-8642. doi: 10.1128/MCB.25.19.8631-8642.2005

Dieci, G., Conti, A., Pagano, A., and Carnevali, D. (2013). Identification of RNA polymerase III-transcribed genes in eukaryotic genomes. BBA-Gene Regul. Mech. 1829, 296-305. doi: 10.1016/j.bbagrm.2012.09.010

Dragovic, Z., Shomura, Y., Tzvetkov, N., Hartl, F. U., and Bracher, A. (2006). Feslp acts as a nucleotide exchange factor for the ribosome-associated molecular chaperone Ssb1p. Biol. Chem. 387, 1593-1600. doi: 10.1515/BC.2006.198

Duttke, S. H. (2014). RNA polymerase III accurately initiates transcription from RNA polymerase II promoters in vitro. J. Biol. Chem. 289, 20396-20404. doi: 10.1074/jbc.M114.563254

Fernández-Bautista, N., Fernández-Calvino, L., Muñoz, A., Toribio, R., Mock, H. P., and Castellano, M. M. (2018). HOP family plays a major role in long-term acquired thermotolerance in Arabidopsis. Plant Cell Environ. 41, 1852-1869. doi: $10.1111 /$ pce.13326

Gibson, D. G. (2011). Enzymatic assembly of overlapping DNA fragments. Methods Enzymol. 498, 349-361. doi: 10.1016/B978-0-12-385120-8.00015-2

Gouge, J., Satia, K., Guthertz, N., Widya, M., Thompson, A. J., Cousin, P., et al. (2015). Redox signaling by the RNA polymerase III TFIIB-related factor Brf2. Cell 163, 1375-1387. doi: 10.1016/j.cell.2015.11.005

Gowda, N. K., Kandasamy, G., Froehlich, M. S., Dohmen, R. J., and Andréasson, C. (2013). Hsp70 nucleotide exchange factor Fes1 is essential for ubiquitindependent degradation of misfolded cytosolic proteins. Proc. Natl. Acad. Sci. U.S.A. 110, 5975-5980. doi: 10.1073/pnas.1216778110

Gowda, N. K., Kaimal, J. M., Masser, A. E., Kang, W., Friedländer, M. R., and Andréasson, C. (2016). Cytosolic splice isoform of Hsp70 nucleotide exchange factor Fes1 is required for the degradation of misfolded proteins in yeast. Mol. Biol. Cell 27, 1210-1219. doi: 10.1091/mbc.E15-10-0697

Gowda, N. K. C., Kaimal, J. M., Kityk, R., Daniel, C., Liebau, J., Öhman, M., et al. (2018). Nucleotide exchange factors Fes1 and HspBP1 mimic substrate to release misfolded proteins from Hsp70. Nat. Struct. Mol. Biol. 25, 83-89. doi: 10.1038/s41594-017-0008-2

Hegemann, J. H., Guldener, U., and Kohler, G. J. (2006). Gene disruption in the budding yeast Saccharomyces cerevisiae. Methods Mol. Biol. 313, 129-144. doi: 10.1385/1-59259-958-3:129

Hoffmann, N. A., Jakobi, A. J., Vorländer, M. K., Sachse, C., and Müller, C. W. (2016). Transcribing RNA polymerase III observed by electron cryomicroscopy. FEBS J. 283, 2811-2819. doi: 10.1111/febs.13732

Hsin, J. P., and Manley, J. L. (2012). The RNA polymerase II CTD coordinates transcription and RNA processing. Genes Dev. 26, 2119-2137. doi: 10.1101/gad.200303.112

Khoo, S. K., Wu, C. C., Lin, Y. C., Lee, J. C., and Chen, H. T. (2014). Mapping the protein interaction network for TFIIB-related factor Brf1 in the RNA polymerase III preinitiation complex. Mol. Cell. Biol. 34, 551-559. doi: 10.1128/MCB.00910-13

Lagrange, T., Hakimi, M. A., Pontier, D., Courtois, F., Alcaraz, J. P., Grunwald, D., et al. (2003). Transcription factor IIb (TFIIB)-related protein (pBrp), a plantspecific member of the TFIIB-related protein family. Mol. Cell. Boil. 23, 32743286. doi: 10.1128/MCB.23.9.3274-3286.2003

Masand, S., and Yadav, S. K. (2016). Overexpression of MuHSP70 gene from Macrotyloma uniflorum confers multiple abiotic stress tolerance in transgenic

\section{SUPPLEMENTARY MATERIAL}

The Supplementary Material for this article can be found online at: https://www.frontiersin.org/articles/10.3389/fpls.2020.00171/ full\#supplementary-material

Arabidopsis thaliana. Mol. Biol. Rep. 43, 53-64. doi: 10.1007/s11033-015-3938-y

Mayer, M. P., and Gierasch, L. M. (2019). Recent advances in the structural and mechanistic aspects of Hsp70 molecular chaperones. J. Biol. Chem. 294, 2085 2097. doi: 10.1074/jbc.REV118.002810

Mclellan, C. A., Turbyville, T. J., Wijeratne, E. M., Kerschen, A., Vierling, E., Queitsch, C., et al. (2007). A rhizosphere fungus enhances Arabidopsis thermotolerance through production of an HSP90 inhibitor. Plant Physiol. 145, 174-182. doi: 10.1104/pp.107.101808

Merret, R., Carpentier, M. C., Favory, J. J., Picart, C., Descombin, J., BousquetAntonelli, C., et al. (2017). Heat shock protein HSP101 affects the release of ribosomal protein mRNAs for recovery after heat shock. Plant Physiol. 174, 1216-1225. doi: 10.1104/pp.17.00269

Saibil, H. (2013). Chaperone machines for protein folding, unfolding and disaggregation. Nat. Rev. Mol. Cell Biol. 14, 630-642. doi: 10.1038/nrm3658

Shen, Y., Chen, X., Peng, B., Chen, L., Hou, J., and Bao, X. (2012). An efficient xylose-fermenting recombinant Saccharomyces cerevisiae strain obtained through adaptive evolution and its global transcription profile. Appl. Microbiol. Biot. 96, 1079-1091. doi: 10.1007/s00253-012-4418-0

Su, P. H., and Li, H. M. (2008). Arabidopsis stromal 70-kD heat shock proteins are essential for plant development and important for thermotolerance of germinating seeds. Plant Physiol. 146, 1231-1241. doi: 10.1104/pp.107.114496

Wang, C., Shen, Y., Zhang, Y., Suo, F., Hou, J., and Bao, X. (2013). Improvement of L-arabinose fermentation by modifying the metabolic pathway and transport in Saccharomyces cerevisiae. BioMed. Res. Int. 2013, 461204. doi: 10.1155/ $2013 / 461204$

Willis, I. M., and Moir, R. D. (2018). Signaling to and from the RNA polymerase III transcription and processing machinery. Annu. Rev. Biochem. 87, 75-100. doi: 10.1146/annurev-biochem-062917-012624

Xing, H. L., Dong, L., Wang, Z. P., Zhang, H. Y., Han, C. Y., Liu, B., et al. (2014). A CRISPR/Cas9 toolkit for multiplex genome editing in plants. BMC Plant Biol. 14, 327. doi: 10.1186/s12870-014-0327-y

Yang, J. Y., Sun, Y., Sun, A. Q., Yi, S. Y., Qin, J., Li, M. H., et al. (2006). The involvement of chloroplast HSP100/ClpB in the acquired thermotolerance in tomato. Plant Mol. Biol. 62, 385-395.

Yi, S. Y., Sun, A. Q., Sun, Y., Yang, J. Y., Zhao, C. M., and Liu, J. (2006). Differential regulation of Lehsp23.8 in tomato plants: Analysis of a multiple stressinducible promoter. Plant Sci. 171, 398-407. doi: 10.1016/ j.plantsci.2006.04.011

Zhang, J. X., Wang, C., Yang, C. Y., Wang, J. Y., Chen, L., Bao, X. M., et al. (2010). The role of Arabidopsis AtFes1A in cytosolic Hsp70 stability and abiotic stress tolerance. Plant J. 62, 539-548. doi: 10.1111/j.1365-313X.2010.04173.x

Zhang, K. Y., Yang, W. W., Yu, H. B., Fu, C., Liu, X. X., and Liu, J. (2019). Double mutation of BRF1 and BRF2 leads to sterility in Arabidopsis thaliana. Biochem. Biophys. Res. Commun. 516, 969-975. doi: 10.1016/j.bbrc.2019.06.138

Zuiderweg, E. R., Hightower, L. E., and Gestwicki, J. E. (2017). The remarkable multivalency of the Hsp70 chaperones. Cell Stress Chaperon. 22, 173-189. doi: 10.1007/s12192-017-0776-y

Conflict of Interest: The authors declare that the research was conducted in the absence of any commercial or financial relationships that could be construed as a potential conflict of interest.

Copyright (c) $2020 \mathrm{Fu}$, Liu, Li, Huo, Ge, Hou, Yang, Zhang, Zhang, Zhao, Ma and Liu. This is an open-access article distributed under the terms of the Creative Commons Attribution License (CC BY). The use, distribution or reproduction in other forums is permitted, provided the original author(s) and the copyright owner(s) are credited and that the original publication in this journal is cited, in accordance with accepted academic practice. No use, distribution or reproduction is permitted which does not comply with these terms. 